\title{
TETRAHIDROTIOFENO: UN LIGANDO POCO CONOCIDO
}

\section{TETRAHYDROTHIOPHENE: A LIGAND NOT VERY FAMOUS}

RESUMEN

\author{
R. Flores-Centurión ${ }^{a}$, J. Santiago Contreras ${ }^{a, b}$
}

Se presenta una revisión de la química del Tetrahidrotiofeno, un ligando poco conocido pero que es utilizado como grupo saliente para generar nuevas moléculas, o unido a metales de transición para generar nuevos complejos.

Específicamente se muestra la utilidad de este ligando en la generación de complejos organometálicos de oro.

Finalmente se presentan los resultados preliminares de la obtención de un complejo de este ligando con oro.

Palabras clave: Tetrahidrotiofeno, complejos, oro.

\section{ABSTRACT}

A review about Tetrahydrothiophene chemistry is presented. This ligand is not very famous but it is used as leaving group to make new molecules, or bonded with transition metals to generate new complexes. Specifically we illustrate the use of this ligand to generate gold organometallic compounds. Finally, the preliminary results about the preparation of a gold(I) complex bonded to Tetrahydrothiophene are presented.

Key words: Tetrahydrothiophene, complexs, gold.

\section{INTRODUCCIÓN}

El tetrahidrotiofeno, THT, es un líquido incoloro, de olor desagradable y altamente tóxico. Por estas razones se le utiliza muy poco en química. Sin embargo tiene propiedades interesantes que pueden ser explotadas, ya sea como solvente, intermediario en la fabricación de medicamentos y pesticidas, como insecticida y como repelente de polillas. También se le utiliza como odorizante de gas y es preferido al etanotiol por su estabilidad térmica, hasta $500^{\circ} \mathrm{C}$, y porque no es corrosivo.

El tetrahidrotiofeno es obtenido a través de una reacción $S_{\mathrm{N}} 2$ entre el 1,4-dicloro butano y el sulfuro de sodio, según el esquema 1:1<smiles>CC(C)(CCCCl)OC(Cl)(Cl)Cl</smiles>

Esquema 1. Síntesis del THT

Es un heterociclo muy estable. La molécula no es plana y presenta un equilibrio de rotación sobre un eje $C_{2}$ (Fig. 1). ${ }^{2}$ La geometría molecular ha sido estudiada por el método ab initio, determinándose un ángulo de torsión de $13^{\circ}$.[3].

La presencia de dos pares libres en el átomo de azufre le confiere al THT propiedades nucleofílicas. Esta propiedad hace que el THT actúe formando aductos relativamente estables. El estudio del enlace de hidrógeno formado entre el THT y el $\mathrm{H}_{2} \mathrm{O}$ ó $\mathrm{HCl}$ muestra que el par libre involucrado se encuentra en un eje casi perpendicular al plano C-S-C del heterociclo (Fig. 2) [4].

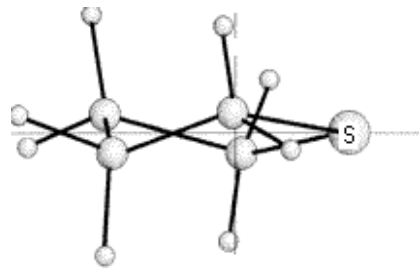

$C_{2}$

Figura 1. Estructura del THT

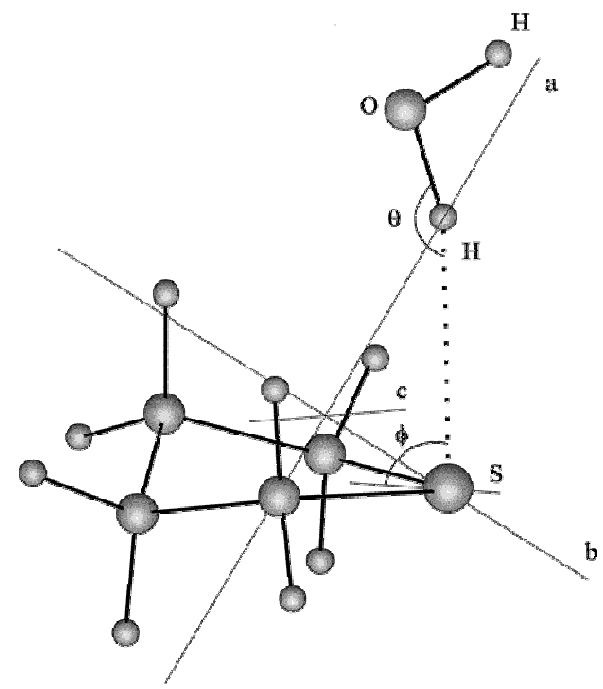

Figura 2. Puente de hidrógeno $\mathrm{THT}-\mathrm{H}_{2} \mathrm{O}$

El THT puede ser producido en el organismo como producto del metabolismo de sustancias como el 1,4dibromobutano $\mathrm{o}$ el dimetilsulfonato de 1,4-butilo (busulfan).[5][6].

La estructura del THT se encuentra como parte de biomoléculas como la biotina, complejo B (Fig. 3). Esta molécula está constituido por un anillo ureido unido al anillo del THT, con el ácido valérico como substituyente.

\footnotetext{
Escuela Académico Profesional de Química, Facultad de Química e Ing. Química, Universidad Nacional Mayor de San Marcos, Lima - Perú

Instituto Peruano de Energía Nuclear, Lima - Perú
} 


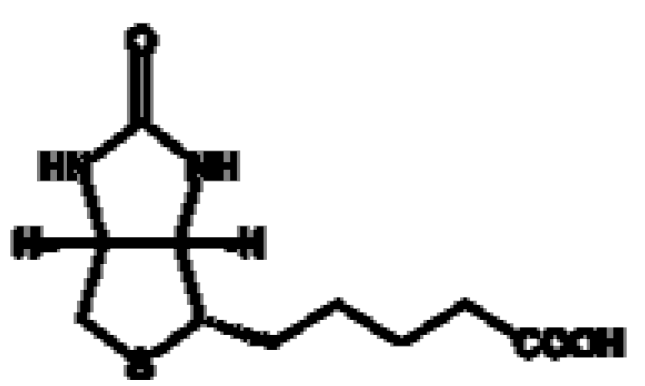

Figura 3. Estructura de la biotina

Los derivados alquilados 2,4- y 2,5-tetrahidrotiofeno poseen actividad biológica, actuando como inhibidores de la enzima 5-lipoxigenasa.

El poli(p-fenilenovinileno), PVV, es un polímero con buenas propiedades para actuar como diodo emisor de luz (LED). Este polímero es preparado a partir de un precursor polimérico con el THT unido como sulfonio. Este grupo sale del polímero gracias a un tratamiento térmico, liberando el THT y formando el PVV (Fig. 4) [7]. También se ha utilizado el THT en la preparación de coloides de oro.[8].

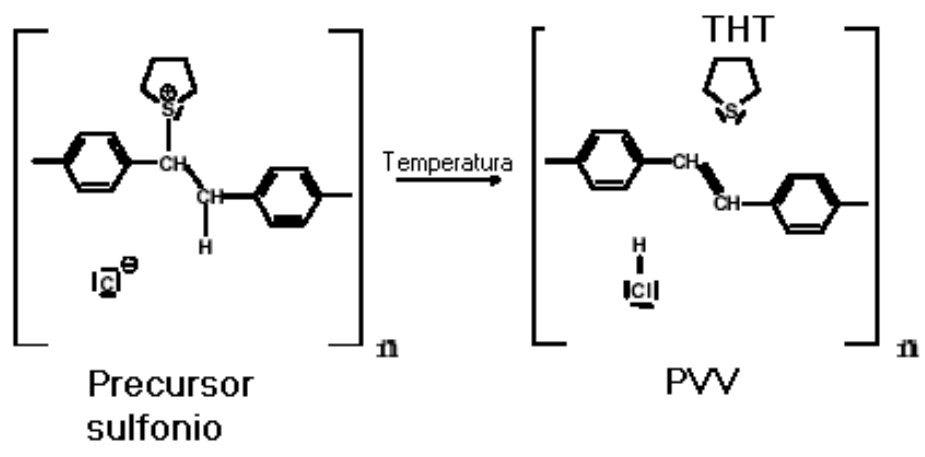

Figura 4. Ejemplo de aplicación del THT como grupo saliente

Debido a la presencia del $\mathrm{S}$, que actúa como átomo donador de densidad electrónica, el THT forma complejos con metales de transición de configuración $d$ [8] como el $\mathrm{Au}(\mathrm{I}), \mathrm{Pd}(\mathrm{II})$ y $\mathrm{Pt}(\mathrm{II})$. En el caso del oro, el compuesto más utilizado para generar toda una serie de compuestos organometálicos es el $\mathrm{Au}$ (tht) $\mathrm{Cl}$. En esta molécula tanto el $\mathrm{Cl}^{-}$como el THT pueden ser reemplazados. $\mathrm{Si}$ se hace reaccionar el $\mathrm{Au}(\mathrm{tht}) \mathrm{Cl}$ con carbaniones se reemplaza el anión $\mathrm{Cl}^{-}$generándose toda una serie de compuestos organometálicos.[9] Se aprovecha el hecho de que el $\mathrm{S}$ se une débilmente a los metales, para reemplazarlo por fosfinas, ${ }^{10,11}$ aminas,[12] o amidas.[13] Todas estas transformaciones tienen que hacerse casi inmediatamente después de preparar el $\mathrm{Au}($ tht $) \mathrm{Cl}$ debido a que este compuesto no es muy estable.

En este trabajo presentamos nuestros resultados preliminares de la preparación del $\mathrm{Au}(\mathrm{tht}) \mathrm{Br}$. Este complejo fue preparado esperando que sea más estable que el $\mathrm{Au}(\mathrm{tht}) \mathrm{Cl}$.

\section{PARTE EXPERIMENTAL}

La preparación del $\mathrm{Au}$ (tht) $\mathrm{Br}$ se realizó en dos etapas. Primero se preparó el $\mathrm{HAuBr}_{4} .2 \mathrm{H}_{2} \mathrm{O}$, por analogía a la preparación del $\mathrm{HAuCl}_{4},[14]$ para luego formar el complejo con el THT.

\section{Síntesis del $\mathrm{HAuBr}_{4} \cdot 2 \mathrm{H}_{2} \mathrm{O}$}

Se disolvió $10 \mathrm{~g}$ de oro en $240 \mathrm{~mL}$ de agua regia. A la solución se agregó $180 \mathrm{~mL}$ de solución acuosa de $\mathrm{HBr}$ $48 \%$. La solución marrón oscuro producía gases pardos. Se dejó reposar por 24 horas y luego se calentó a $80^{\circ} \mathrm{C}$ hasta que salieran gases. En seguida se agregó $1 \mathrm{~mL}$ de $\mathrm{HBr} 48 \%$. Este proceso se repitió 4 veces. Finalmente, los cristales fueron lavados con etanol.

\section{Síntesis del $\mathrm{Au}(\mathrm{tht}) \mathrm{Br}$}

En un balón de 3 bocas, provisto de una pera de adición, refrigerante y termómetro, se agregó $1 \mathrm{~g}$ de $\mathrm{HAuBr}_{4} .2 \mathrm{H}_{2} \mathrm{O}, 40 \mathrm{~mL}$ de EtOH y $1 \mathrm{~mL}$ de agua. Esta solución fue calentada a $50^{\circ} \mathrm{C}$ y luego se le agregó, lentamente y bajo agitación, una solución de $2 \mathrm{~mL}$ de THT disuelto en $10 \mathrm{~mL}$ de EtOH. Al término de la adición del THT se continuó calentando por 20 minutos adicionales. Al enfriarse la solución se formaron cristales blancos en forma de aguja, que fueron lavados con dietiléter y etanol. Análisis elemental: (calculado) C, 13.45 (13.16); H, 2.3 (2.21) y S, 8.0 (8.78).

\section{RESULTADOS Y DISCUSIÓN}

El complejo $\mathrm{Au}$ (tht) $\mathrm{Br}$ ha sido caracterizado por sus espectros ${ }^{1} \mathrm{H}$-RMN y por FT-IR. El espectro de RMN del protón presenta cuatro señales. Las señales a 7.24 y 1.60 ppm corresponden al solvente, $\mathrm{CDCl}_{3}$, y agua respectivamente. Las señales a $2.20\left(\mathrm{CH}_{2}-\mathrm{CH}_{2}-\mathrm{S}\right)$ y 3.42 ppm $\left(\mathrm{CH}_{2}-\mathrm{S}\right)$ corresponden a los grupos metileno del THT, pero no se observa el triplete esperado para cada par de $\mathrm{CH}_{2}$. El ligando libre presenta señales a campos más altos, 1.94 y 2.82 ppm respectivamente. Esta diferencia se debe a la interacción del metal con el átomo de azufre del THT. La forma ancha de estos picos hace pensar de que hay elementos paramagnéticos, sin embargo el $\mathrm{Au}(\mathrm{I})$ es diamagnético. Sospechamos que el compuesto se ha descompuesto parcialmente dando lugar a especies paramagnéticas. Esto es posible pues se sabe que el $\mathrm{Au}(\mathrm{I})$ tiene tendencia a la dismutación. Efectivamente, se ha observado que este compuesto es sensible a la luz, oscureciéndose rápidamente. Luego de 4 días se descomponían en aprox. 50\%. Otra posible explicación es el poder oxidante del precursor $\mathrm{HAuBr}_{4}$ que oxida al THT, reduciendo el estado de oxidación del metal a $\mathrm{Au}(\mathrm{I})$, pudiendo llegar a oro metálico. Sin embargo, se ha utilizado un gran exceso de este solvente para evitar que el Au se reduzca hasta Au metálico, lo cual sucede cuando se quiere preparar oro metálico coloidal. ${ }^{15}$ El exceso de THT permite que el Au estabilice el estado de oxidación +1 por la formación del complejo deseado. Es probable que, a pesar del cuidado en las manipulaciones, se haya obtenido un muy pequeño porcentaje de oro coloidal, suficiente para producir el ensanchamiento de las señales en el espectro RMN. 
El análisis del espectro IR no nos permite identificar el enlace $\mathrm{Au}-\mathrm{Br}$ pues está fuera de la ventana permitida por el equipo utilizado. El valor teórico para este enlace es de $220 \mathrm{~cm}^{-1}$. Sin embargo se observan los picos del ligando libre ligeramente desplazados por efecto de la influencia del metal.

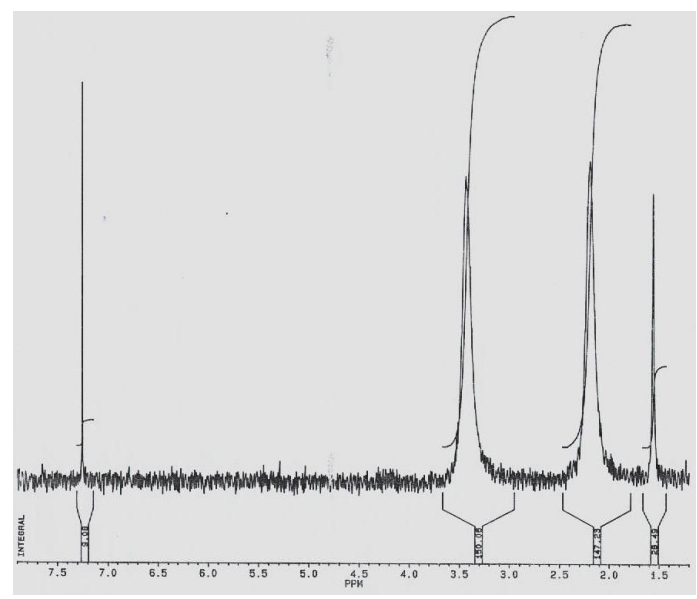

Figura 5. Espectro [1] H-RMN del $\mathrm{Au}$ (tht) $\mathrm{Br}$

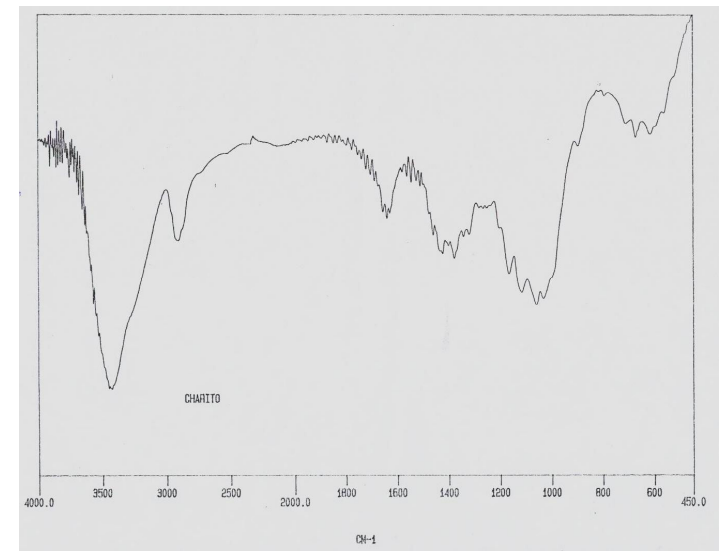

Figura 6. Espectro IR del $\mathrm{Au}($ tht $) \mathrm{Br}$

Los valores obtenidos en el análisis elemental del $\mathrm{Au}$ (tht)Br son cercanos a los valores teóricos, excepto para el azufre. Estos valores permiten descartar la posibilidad de que se haya obtenido el complejo $\mathrm{Au}$ (tht) $\mathrm{Br}_{3}$, tal como sucede durante la síntesis del $\mathrm{Au}$ (tht)Cl.
A pesar de que el oro(I) tiene su última capa electrónica completamente llena ([Xe] 4f[14] 5d[10]), se estabiliza mejor formando complejos lineales al igual que los otros cationes monovalentes del mismo grupo, $\mathrm{Cu}(\mathrm{I})$ y $\mathrm{Ag}(\mathrm{I})$. La razón de este comportamiento es la formación dos orbitales híbridos como producto de la hibridación de los orbitales $n s, n p$ y $(n-1) d$. Este tipo de hibridación es posible por la semejanza energética de dichos orbitales. Todos estos cationes son considerados blandos, según la terminología de Pearson, por la diferencia relativamente pequeña entre los orbitales HOMO y LUMO. Se trata de un catión blando que tiene buena afinidad por aniones blandos, es decir voluminosos y de baja carga o neutros. Por dicha razón, la afinidad por los aniones haluro sigue el siguiente orden: $\mathrm{I}^{-}>\mathrm{Br}^{-}>\mathrm{Cl}^{-16}$

\section{CONCLUSIÓN}

El tetrahidrotiofeno es una molécula con propiedades que le permiten unirse a otras a través del átomo de azufre. Este enlace es relativamente débil, lo cual es explotado en la preparación de nuevas moléculas orgánicas, complejos de transición u com- puestos organometálicos. Tiene una buena afinidad por los metales de configuración d8. Esta propiedad es muy explotada en la química del oro.

\section{REFERENCIAS BIBLIOGRÁFICAS}

Los resultados preliminares de esta investigación nos dan evidencia importan- te acerca de la preparación del complejo Au(tht)Br. Sin embargo, quedan aún cuestiones por aclarar.

\section{AGRADECIMIENTOS}

Se agradece a la DAAD (Servicio alemán de intercambio académico) por el financiamiento de R. Flores para realizar este trabajo en la Universidad de Braunschweig, Alemania. 


\section{REFERENCIAS BIBLIOGRAFICAS}

[1]. Lawson J., Easley W., Wagner W., Organic Syntheses, Coll. Vol. 36, 89.

[2]. Sanz M., López J., Alonso J., J. Phys. Chem. A, 102, 3681(1998).

[3]. El-Gogary T., Spectrochim. Acta A Mol. Biomol. Spectrosc., 57, 1405(2001).

[4]. Sanz M., López J., Alonso J., J. Phys. Chem. A, 103, 5285(1999).

[5]. Onkenhout W., van Loon W., Buijs W., van der Gen A. , Vermeulen N., Drug metab. Dispos., 14, 608(1986).

[6]. Ritter C., Sperker B., Grube M., Dressel D., Kunert-Keil C., Kroemer H., British J. Pharm., 137, 1100(2002).

[7]. Crispin X., Crispin A., de Jong M., Marciniak S., Osikowicz W., Jönsson S., Fahlman M., Kugler Th., van IJzendoorn L., de Voigt M., Salaneck W., Mat. Res. Soc. Symp. Proc., 747, V5.5.1(2003).

[8]. Duff D., Baiker A., Edwards P., Langmuir, 9, 2301(1993).

[9]. Usón R., Laguna A., Vicente J., J. Chem. Soc., Chem. Commun., 353(1976).

[10]. Gimeno M., Laguna A., Gold Bulletin, 32, 90(1999).

[11]. Leznoff D., Rancurel C., Sutter J., Rettig S., Pink M., Paulsen C., Kahn O., J. Chem. Soc., Dalton Trans., 3593(1999).

[12]. Ahrens B., Jones P., Fischer A., Eur. J. Inorg. Chem., 7, 1103 (1999).

[13]. Mohamed A., Abdou H., Irwin M., López-de-Luzuriaga J., Fackler J., J. Cluster Sci., 14, 253(2003).

[14]. Usón R., Laguna A., Organomet. Synth., 3, 322(1986).

[15]. Sun X., Dong S., Wang E., Chem. Commun., 1182(2004).

[16]. Shriver D., Atkins P., Langford C., Química Inorgánica, Ed. Reverté, Vol. 1, Barcelona, 353, 1998.

E-mail: charoflores@yahoo.es 